\title{
Facial Feature Extraction Based on Weighted ALW and Pulse-Coupled Neural Network
}

\author{
Junhua Liang ${ }^{1}$, Zhisheng Zhao ${ }^{1 *}$, Xiao Zhang ${ }^{1}$, Xuan Wang ${ }^{2}$ and Yang Liu ${ }^{1}$ \\ ${ }^{1}$ School of Information Science and Engineering, Hebei North University, \\ Zhangjiakou 075000, Hebei, China \\ ${ }^{2}$ School of Physics and Information Technology, Shaanxi Normal University, \\ $X i$ 'an 710062, Shaanxi, China \\ 1010651711@qq.com
}

\begin{abstract}
In order to improve the robustness of face identification with the changes of illumination, expression and facial alteration, a new facial feature extraction algorithm based on weighted adaptive lifting wavelet(ALW) scheme and pulse-coupled neural network $(P C N N)$ is involved in this paper. The face images are decomposed into several subbands by weighted adaptive lifting scheme. Then the PCNN is utilized to decompose each weighted subbands into a series of binary images, the entropies of which are calculated and regarded as facial features. Experimental results show that the method yields a good robustness against the illumination, expression and facial variability and reduces the computer burden.
\end{abstract}

Keywords: PCNN; adaptive lifting wavelet; face recognition

\section{Introduction}

Face recognition possesses important theoretical research value as a typical research topic in image processing, pattern recognition, and artificial intelligence. Simultaneously, it has broad application prospects in financial security, criminal detection, public security and other fields [1].

Feature extraction algorithm is the main step of face recognition which attracts great attention of scholars [2]. With the change of illumination, expression, posture and facial variation, robustness and rapidity of feature extraction are the key problems in face recognition application [2]. Existing methods for facial feature extraction are classified into four methods including: geometric-based, model-based, subspace-based and spacialfrequency domain-based [3]. Geometric-based method mainly extracts the geometric structure of major facial organs, however, if certain expression or posture changes, the identification accuracy is poor. In order to overcome the above problems, the method based on transform domain or model is proposed. Face image will be decomposed into several subbands, the high frequency of which is considered as feature in Gabor, wavelet and Contourlet transform [4-6]. The Markov or Hidden Markov model is utilized to simulate the statistical characteristics of input signal while the face image is considered as a random variable [7]. These methods partly improve the robustness of expression, posture and illumination changes. But it can't meet the real-time requirement because of its high computation complexity. Face image will be projected into subspace by subspace methods [8], such as principal component analysis (PCA), linear decision analysis [9-10] (LDA) and independent component analysis (ICA). The projection coefficient is considered as feature. The method has higher computation efficiency, but the recognition rates

*Corresponding Author 
and robustness will decline with the change of expression, posture and position, because it can hardly distinguish the differences caused by the external environment and face image itself.

Recently, several frameworks of lifting wavelet transform were applied into face feature extraction [11], which inherited the advantages of multiresolution representation over the traditional wavelet transform such as flexible design, low computational complexity and real-time application. According to the discontinuities playing an important role in face image, we put forward a modified adaptive lifting scheme to protect the edge information, and adapt the weighted subbands considering the different contribution each subband takes in the whole image. In this paper, weighted adaptive lifting scheme is utilized to decompose the face image into several subbands, then the weight of each subband is computed, and will be sent to Pulse-coupled Neural Network as input, through which we can obtain a series of binary images, the entropies of these binary images are considered as facial feature. The experimental results in ORL and YALE face database show that the algorithm is efficient contrast to subspace method, while it obtains strong robustness against the change of light, posture and facial expression.

\section{Lifting Wavelet Scheme and Pulsed-Coupled Neural Network}

\subsection{Lifting Scheme}

The lifting wavelet scheme called the second generation wavelet transform provides us with a simple explanation of the basic theory of wavelet method [14]. In lifting wavelet scheme, transformation process is divided into three steps: split, predict and update. The decomposition and reconstruction are just shown in Figure 1.

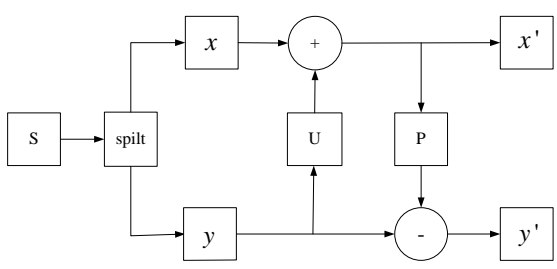

(a)

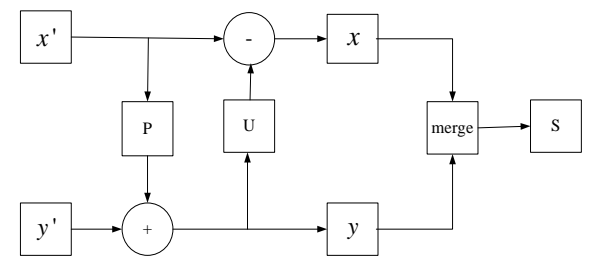

(b)

Figure 1. Lifting Scheme (a) and Reconstruction (b)

Spilt: The original signal $s$ is divided into low frequency ${ }^{x}$ and high frequency ${ }^{y}$ by a particular transform such as the simple polynomial decomposition.

Update: An update map ${ }^{U}$ will act on the high frequency ${ }^{y}$ to modify ${ }^{x}$ yielding a new low frequency ${ }^{x}$, i.e.,

$x^{\prime}=x+U(y)$

Predict: A prediction map ${ }^{P}$ operating on ${ }^{x}$ is used to modify ${ }^{y}$ resulting in a new high frequency ${ }^{y}$, i.e.,

$y^{\prime}=y-P\left(x^{\prime}\right)$

Actually, the reconstruction process is the reversed lifting scheme steps as shown in Figure 1 (b). From the above operations we can see that the lifting wavelet achieved situ operation, in other words, the old data can be replaced with new data flow stream at each data point, thereby a lot of storage space is saved by lifting scheme. In these way it can avoid complex computation resulted from the repeated convolution, meanwhile real-time performance is necessary for FPGA realization. 


\subsection{Pulse-Coupled Neural Network}

Pulse-coupled Neural Network is proposed by Johnson, according to neurons sync pulse phenomenon in cat visual cortex. PCNN is invariant to rotation, scaling and distortion changes [12-13], which is composed of receiving unit, connecting unit and pulse generator. In image processing, each pixel and neuron are corresponding to each other. For better application, this paper adopts the following representation of neural network model [15]:

$$
\begin{aligned}
& F_{i j}(n)=S_{i j} \\
& L_{i j}(n)=\sum_{k l} W_{i j k l} Y_{k l}(n-1) \\
& U_{i j}(n)=F_{i j}(n)\left(1+\beta L_{i j}(n)\right) \\
& X_{i j}(n)=\frac{1}{1+\exp \left(E_{i j}(n-1)-U_{i j}(n)\right)} \\
& Y_{i j}(n)=\left\{\begin{array}{l}
1, \quad X_{i j}(n)>0.5 \\
0, \quad \text { else }
\end{array}\right. \\
& E_{i j}(n)= \begin{cases}V_{E}, & Y_{i j}(n)=1 \\
\alpha_{E} E_{i j}(n-1) \quad Y_{i j}(n)=0\end{cases}
\end{aligned}
$$

In which, $n$ is the current iteration, $(i, j)$ represents the neuron location, $S$ is input, $M$ and $W$ are called weight matrix, $V_{L}, V_{F}$ and $V_{T}$ indicate the inherent voltage respectively, $\beta$ is the connecting coefficient, $\alpha_{F}, \alpha_{L}$ and ${ }^{\alpha_{T}}$ represent attenuation time constant. The model is a simplification over standard Pulse Coupled Neural Network, which should only set $V_{E}, \alpha_{E}$ and $\beta$ avoiding a lot of computation burden caused by preset parameters. These undetermined parameters will be different in application fields, so there is no general theoretical method except conducting experiment.

\section{The Proposed Algorithm}

\subsection{Weighted Lifting Wavelet Scheme}

Classical lifting scheme, where the update map $\mathrm{U}$ and the prediction map $\mathrm{P}$ are fixed, obviously adopts the uniform smooth operation for the whole image, which hardly precisely locates the region of interest or discontinuities. In this section we build a weighted lifting wavelet scheme taking full account of the alternating details.

Spilt: The original image $f(i, j)$ with size $N \times M$ is split into four images $x(n, m) y_{h}(n, m) y_{v}(n, m) y_{d}(n, m)$ with size $N / 2 \times M / 2$ by the simple polyphase decomposition, shown as Figure 2: 

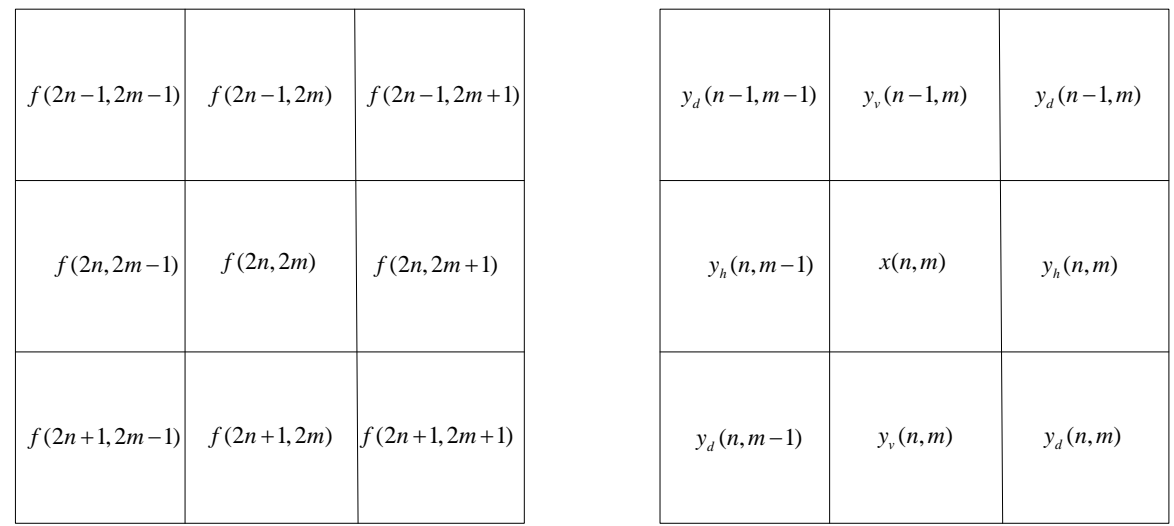

Figure 2. Left: Coordinates for Two-Dimensional Signal. Right: Location of the Input $x(n, m) \quad y_{h}(n, m) \quad y_{v}(n, m) \quad y_{d}(n, m)$ after Polyphase Decomposition

Prediction: the output approximation signal $x^{\prime}(n, m)$ equals

$x^{\prime}(n, m)=x(n, m) \oplus_{p_{n m}} U_{p_{n m}}\left(y_{h}(n, m), y_{v}(n, m), y_{d}(n, m)\right)$

where ${ }^{p_{n m}}$ is the output of the binary decision map $D$, that is

$p_{n m}=D(n . m)= \begin{cases}0 & \sigma(n, m)>T \\ 1 & \sigma(n, m) \leq T\end{cases}$

where $T$ is a given threshold, and $\sigma(n, m)$ is defined as

$\begin{aligned} \sigma(n, m)= & \left|y_{h}(n-1, m)-\mu\right|+\left|y_{h}(n, m)-\mu\right|+\left|y_{v}(n, m-1)-\mu\right|+\left|y_{v}(n, m)-\mu\right| \\ & +\left|y_{d}(n-1, m)-\mu\right|+\left|y_{d}(n, m)-\mu\right|+\left|y_{d}(n, m-1)-\mu\right|+\left|y_{d}(n-1, m-1)-\mu\right|\end{aligned}$

where ${ }^{\mu}$ denotes the mean of the eight horizontal, vertical and diagonal neighbors of $x(n, m)$, i.e.,

$\mu=\frac{1}{8}\left[y_{h}(n-1, m)+y_{h}(n, m)+y_{v}(n, m-1)+y_{v}(n, m)+y_{d}(n-1, m)\right.$

$\left.+y_{d}(n, m)+y_{d}(n, m-1)+y_{d}(n-1, m-1)\right]$

In our adaptive lifting scheme, ${ }^{p_{n m}}$ governs the choice of the update step. For every possible decision ${ }^{p_{n m}}$, we have a different addition operator ${ }^{\oplus}{ }_{p_{n m}}$ and update operator $U_{p_{n m}}$. In other words, they are location-dependent and adaptive. Update operator $U_{p_{n n}}$ is given by

$$
U_{p_{n m}}= \begin{cases}0 & p_{n m}=0 \\ \frac{1}{4}\left[y_{h}(n-1, m)+y_{h}(n, m)+y_{v}(n, m-1)+y_{v}(n, m)\right] & p_{n m}=1\end{cases}
$$

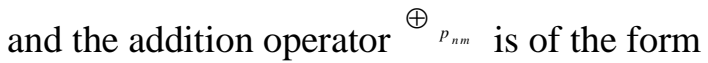


$x \oplus_{p_{n m}} u= \begin{cases}x+u & p_{n m}=0 \\ \frac{1}{2}(x+u) & p_{n m}=1\end{cases}$

As shown in Figure 5, in our adaptive lifting scheme, the update map $\mathrm{U}$ is adaptive, while the prediction step is fixed. The prediction operators $P_{h}\left(x^{\prime}(n, m)\right), P_{v}\left(x^{\prime}(n, m)\right)$ and $P_{d}\left(x^{\prime}(n, m)\right)$ are defined as follows:

$P_{h}\left(x^{\prime}(n, m)\right)=\frac{1}{2}\left[x^{\prime}(n-1, m)+x^{\prime}(n, m)\right]$

$P_{v}\left(x^{\prime}(n, m)\right)=\frac{1}{2}\left[x^{\prime}(n, m-1)+x^{\prime}(n, m)\right]$

$P_{d}\left(x^{\prime}(n, m)\right)=\frac{1}{2}\left[x^{\prime}(n-1, m-1)+x^{\prime}(n, m)\right]$

The detail signals $y_{h}^{\prime}(n, m), y_{v}^{\prime}(n, m)$ and $y_{d}^{\prime}(n, m)$ are calculated by

$y_{h}^{\prime}(n, m)=y_{h}(n, m)-P_{h}\left(x^{\prime}(n, m)\right)$

$y_{v}^{\prime}(n, m)=y_{v}(n, m)-P_{v}\left(x^{\prime}(n, m)\right)$

$y_{d}^{\prime}(n, m)=y_{d}(n, m)-P_{d}\left(x^{\prime}(n, m)\right)$



Figure 3. Our 2-D Adaptive Lifting Scheme

The contribution of each detail signal to the entire image is different, which can be computed as follows:

$\alpha_{i}=\frac{\sum x_{i}(n, m)}{\sum_{m=1}^{M} \sum_{n=1}^{N} f(n, m)}$

The basic idea underlying our adaptive scheme is stated as follows: for smooth regions where $p_{n m}=1$, we compute $x^{\prime}(n, m)$ as the weighted average of $x(n, m)$ and its eight horizontal, vertical and diagonal neighbors, whereas for less homogeneous regions where 
$p_{n m}=0$, we do not perform any filtering, i.e., $x^{\prime}(n, m)=x(n, m)$. The main reason to do this is that the discontinuities in face images play an significant role in face identification, but there are many isolated discontinuous points resulted from noise or other distortions, which may disturb face identification, and should be filtered. By adaptive update and prediction, we can extract the face information with filtering the distortions. In addition, our adaptive scheme only involves the addition and subtraction operations in spatial domain, and holds high parallel property, in situ operation, so it presents very low computational cost and easy hardware implementation. Figure 4 shows the decomposition results (at level 1) via our weighted adaptive scheme and the wavelet transform used in [11]. It is noted from the detailed images in Figure 4 that the weighted adaptive scheme preserves the edges well with less distortions in comparison with the wavelet transform.
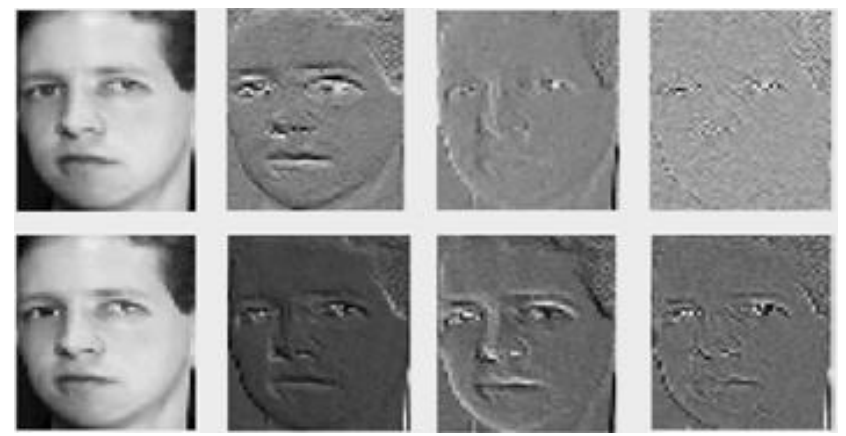

Figure 4. The Decomposition Results (At Level 1) Using Our Weighted Adaptive Scheme and the Wavelet Transform Used in [11], Top: (From Left to Right): The Approximate, Horizontal, Vertical and Diagonal Detail Images of the Wavelet Transform. Bottom (From Left to Right): The Approximate, Horizontal, Vertical and Diagonal Detail Images of Our Weighted Adaptive Scheme

\subsection{The Proposed Method}

Face image is always influenced by some factors such as facial expression, illumination and facial modification, which will reduce the recognition and limit its application. Meanwhile, real-time is a key requirement in practice, so it is necessary to look for a strongly robust and real-time algorithm.

The characteristics obtained by weighted adaptive lifting wavelet is not sensitive to expression change, and fully take into account of contour edge plays a key role in face recognition. Adaptive lifting wavelet outperforms because of flexible design and low computational complexity. Pulse coupled neural network itself has the invariant characteristics against the rotation, scale and translation meanwhile it can better explain the global features of the image. It will get stronger robustness combining the local information by weighted adaptive lifting wavelet and global information by pulse coupled neural network.

The low frequency component in face image represents most information, describing the invariant features of face. It is proved that approximation component is best for recognition at the first layer decomposition in literature [16]. Therefore, this article choose the first layer of low frequency component as the feature, greatly reducing the complexity of calculation. The high frequency component in face image respresent details corresponding to illumination, facial expression and location change. Statistical results show that the diagonal component contains too many local informations caused by illumination, rotation, and facial expression change, while the excessive internal pattern changes will affect the recognition performance. Considering the recognition performance and computational 
complexity, we select the horizontal component and vertical component at one layer as the feature.

The implementation steps in feature extraction process are as follows:

Step 1: Get approximate, horizontal and vertical subbands by weigthed adaptive lifting wavelet at the first layer for a given face image.

Step 2: The decomposed subbands will be considered as the input of pulse coupled neural network, simulating the perception process of biological visual cortex, and converted into a series of cognition sequence of binary image.

Step 3: The entropy of each binary sequence is calculated and considered as the final classification recognition characteristics.

After several test experiments, the three input parameters with PCNN model in our feature extraction process are shown in Table 1. Form the table, the inherent potential of dynamic threshold $V_{E}$ is set as half of the biggest grey values of each image. The decay time constant $^{\alpha_{E}}$ and the strength connection coefficient $\beta$ is selected the optimal value on the basis of many experiments, which get better effect in ORL and YALE face database.

Table 1. The Parameters of PCNN

\begin{tabular}{ccc}
\hline$V_{E}$ & $\alpha_{E}$ & $\beta$ \\
\hline 127.5 & 0.8 & 0.03 \\
\hline
\end{tabular}

Connected weighted coefficient matrix $M$ and $W$ set like this:

$$
M=W=\left[\begin{array}{ccc}
0.5 & 1 & 0.5 \\
1 & 0 & 1 \\
0.5 & 1 & 0.5
\end{array}\right\rfloor
$$

The subbands decomposited by weighted adaptive lifting wavelet are converted to a series of binary cognition sequences through PCNN model which maybe different because of different gray value distribution. So we calculate the entropy of the binary sequence as final feature as that. Shannon entropy has the scaling, translation and rotation invariant features. The entropy is defined as:

$$
H(p)=-p_{0} \log \left(p_{0}\right)-p_{1} \log \left(p_{1}\right)
$$

Where ${ }^{p_{0}}$ and ${ }^{p_{1}}$ respectively represent the probability of pixels 0 and 1 in binary sequence. The introduction of entropy and the combination of local special processing using weighted adaptive lifting wavelet with rotation, scaling, distortion invariant nature of pulse coupled neural network [13], up to the design requirements of face recognition robustness.

\section{Simulation and Analysis}

\subsection{Experiment in ORL}

Our experimental simulation environment is Intel(R) Celeron(R) CPU $2.1 \mathrm{GHz}, 2 \mathrm{~GB}$ memory, Microsoft Windows XP professional system, and Matlab7.0 software. ORL face database contains 256 gray values corresponding to 400 different facial images, the resolution of each image is $92 * 112$. The 10 face images of each person include different facial expression change, tiny attitude change, and less than 20\% scale change. Figure 5 shows the facial expressions of two person random select in ORL face database. There is no any preprocessing for the test face image in our experimental process. 


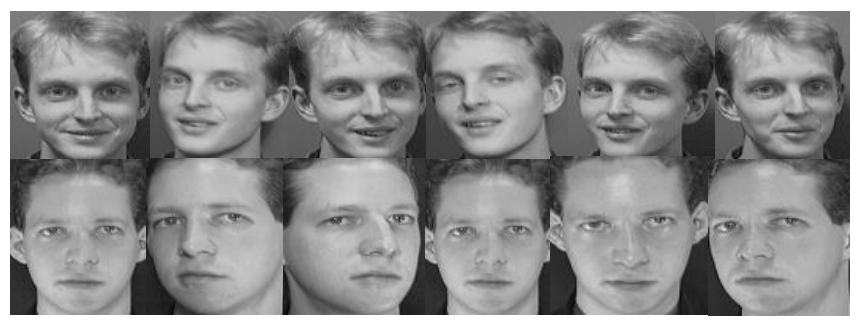

\section{Figure 5. Two Person's Facial Expression Images Random Selected from ORL Face Database}

We respectively random select 3,4 , and 5 , samples in ORL face database as training set, the remaining as test set. Our algorithm is compared with the principal component analysis (PCA) [8], linear decision analysis (LDA) [9], independent component analysis (ICA) [10], M-PCNN [15] and the algorithm in literature [17]. In order to eliminate the influence of different classifier for recognition accuracy, each method is classified by support vector machine with the optimum parameters. All kinds of algorithms take the average recognition accuracy of 20 times. The contrast results of robustness are shown in Table 2 in terms of variance which respresent the deviation of each training.

From Table 2, the robustness of our algorithm is significantly higher than the classic subspace method and only PCNN method. In addition, the method of literature [17] has been carried on the comparison which combines the visual perception with edge protection. It performs slight difference between the two methods.

Table 2. The Recognition Performance in Terms of Identify Precision (\%) and Variance in ORL Face Database

\begin{tabular}{|c|c|c|c|c|}
\hline \multirow[t]{2}{*}{ Method } & \multicolumn{3}{|c|}{ Recognition rate } & \multirow[t]{2}{*}{$\begin{array}{l}\text { Vari- } \\
\text { ance }\end{array}$} \\
\hline & $\begin{array}{c}\text { 3Trai } \\
n\end{array}$ & $\begin{array}{c}\text { 4Trai } \\
\mathrm{n}\end{array}$ & $\begin{array}{c}5 \text { Trai } \\
n\end{array}$ & \\
\hline PCA & 78.52 & 82.08 & 87.5 & 6.3951 \\
\hline LDA & 83.57 & 85.41 & 92.00 & 6.2684 \\
\hline ICA & 83.21 & 83.75 & 91.00 & 6.1519 \\
\hline M-PCNN & 85.35 & 87.91 & 94.50 & 6.6759 \\
\hline $\begin{array}{c}\text { Litera- } \\
\text { ture[17] }\end{array}$ & 91.02 & 92.50 & 99.30 & 6.0415 \\
\hline Our method & 91.07 & 95.83 & 99.49 & 5.9708 \\
\hline
\end{tabular}

\subsection{Experiment in YALE}

It includes 165 frontage face images from 15 samples with the resolution of $100 * 100$ and 256 grayscale values in YALE face database. These sample images are obtained under more obvious light condition, gesture and facial expression [18], which perform as high as 200 degree of depth rotation and plane rotation even ten percent of face scale change. Figure 6 shows two person's face image randomly selected in YALE face database. It can further verify the robustness of these algorithms because of more rich facial expression, uneven illumination change, and sunglasses wearing. 


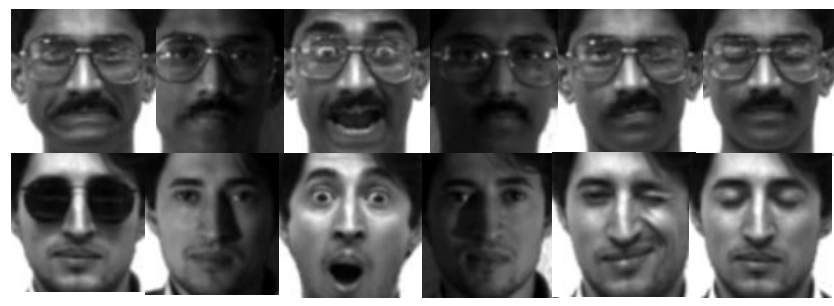

\section{Figure 6. Two Person's Facial Expression Images Randomly Selected in} YALE Face Database

This experiment randomly select $3,4,5$, sample images of each person as training, the rest for test. Classifier uses support vector machine (SVM) with the optimization parameters. The identification accuracy and variance of all kinds of algorithms are shown in Table 3, as you can see from the table, all recognition rates of these algorithms are decreased, mainly due to the obvious illumination, posture, and accessories change in YALE face database. But the robustness of our method is significantly higher than that of the classical PCA and LDA algorithm, and more stability than the single M-PCNN method. The decline degree of robustness and variance is close to the literature [17] method.

Table 3. The Recognition Performance in Terms of Identify Precision (\%) and Variance in YALE Face Database

\begin{tabular}{|c|c|c|c|c|}
\hline \multirow[t]{2}{*}{ Method } & \multicolumn{3}{|c|}{ Recognition Rate } & Vari- \\
\hline & 3Train & 4Train & 5Train & \\
\hline PCA & 63.08 & 70.38 & 73.11 & 9.2573 \\
\hline LDA & 71.08 & 81.43 & 83.89 & 9.6137 \\
\hline ICA & 61.50 & 68.70 & 71.40 & 9.1558 \\
\hline M-PCNN & 79.30 & 84.80 & 92.70 & 9.5257 \\
\hline $\begin{array}{l}\text { Litera- } \\
\text { ture[17] }\end{array}$ & 82.16 & 88.69 & 93.28 & 7.9028 \\
\hline Our method & 82.23 & 87.78 & 93.33 & 7.8489 \\
\hline
\end{tabular}

Due to the demand of real-time performance and hardware realization in practice, the compute cost of feature extraction time is compared just as shown in Table 4. From the perspective of real-time analysis, our features only contain three subbands with only one layer. Compared with the tens of thousands of dimension in original image, our method reduces nearly two orders of magnitude, and greatly reduce the time complexity, significantly lower than the literature [17] and traditional subspace algorithm. Meanwhile adaptive lifting wavelet can meet the requirements of hardware implementation because of situ operation which greatly save the storage space.

Table 4. Feature Extraction Time (ms) of These Compared Method

\begin{tabular}{ccccccc}
\hline Methods & $\begin{array}{c}\text { PC } \\
\text { A }\end{array}$ & LDA & ICA & M-PCNN & $\begin{array}{c}\text { Litera- } \\
\text { ture[17] }\end{array}$ & Our method \\
\hline $\begin{array}{c}\text { Extraction } \\
\text { time }\end{array}$ & $\begin{array}{c}3.4 \\
1\end{array}$ & 3.75 & 3.48 & 3.19 & 4.34 & 3.05 \\
\hline
\end{tabular}

\section{Conclusion}

In order to improve the robustness of illumination, posture, facial expression change in face recognition, and to meet the requirement of real-time performance and calculation 
cost in practical application, a weighted adaptive lifting scheme combined with pulse coupled neural networks is proposed in this paper. This method combines the local detail processing operation in weighted adaptive lifting scheme with the scaling, translation and rotation invariant advantages in pulse coupled neural networks, which yields strong robustness and low calculation cost in the contrast experiment of ORL and YALE face database. Considering the requirements of robustness and calculate burden, our algorithm perform high effectiveness.

\section{Acknowledgements}

This work was supported by Hebei Province Population Health Information Engineering Technology Research Center, the Funding Project of Science \& Technology Research and Development in Hebei North University (Grant No. ZD201301), Major Scientific Research Projects in Higher School of Hebei Province (Grant No. ZD20130850), Major Projects in Hebei Food and Drug Administration (Grant No.ZD2015017), Major Research Projects in Hebei HealthDepartment (Grant No. ZL20140127), Youth fund projects of science and technology in Higher School of Hebei Province .

\section{References}

[1] N. Kwaka, "Feature extration for classification problems and its application to face recognition", Pattern Recognition, vol. 41, no. 5, (2008).

[2] L. Qiao, S. Chen and X. Tan, "Sparsity preserving projections with applications to face recog-nition", Pattern Recognition, vol. 43, no. 1, (2010).

[3] R. Jafri and H. R. Arabnia, "A survery of face recognition rechniques”, Journal of inf-ormation processing systems, vol. 5, no. 2, (2009)

[4] M. Yang and L. Zhang, "Gabor feature based robust representation and classification for face recognition with Gabor occlusion dictionary”, Pattern Recognition, vol. 46, no. 7, (2013).

[5] B. Xu and H. Y. Li, "Wavelet Face Recognition Using Bayesian Classifier", Advanced Materials Research, (2012).

[6] Y. C. He, W. B. Liu and G. Zhang, "Rotation-invariant texture image retrieval algorithm based on nonsubsampled contourlet transform", Journal of Image and Graphics, 1.6.(2011).

[7] Wang ZH CH, Liu H Y.A face recognition based on hidden markov model. Computer Applications and Software, vol. 30, no. 2, (2013).

[8] N. L. Shchegoleva and G. A. Kukharev, "Application of two-dimensional principal component ana-lysis for recognition of face images", Pattern Recognition and Image Analysis, vol. 20, no. 4, (2010).

[9] H. Yu and J. Yang, "A direct LDA algorithm for high-dimensional data with application to face recognition", Pattern Recognition, vol. 34, no. 10, (2000).

[10] M. S. Bartlett, J. R. Movellan and J. Sejnowskit, "Face Recognition by Independent Component Analysis", IEEE Transaactions on Neural Networks, vol. 13, no. 6, (2002).

[11] X. Wang and J. H. Liang, "On-line fast palmprint identification based on adaptive lifting wavelet scheme”, Knowledge-Based Systems, vol. 42, (2013).

[12] J. L. Johnson and D. Ritter, "Observation of periodic waves in a Pulse-coupled neural network", Optics Letters, vol. 18, no. 15, (1993).

[13] J. L. Johnson, "Pulse-coupled neural nets:Translation, rotation, scale, distortion and intensity signal invariance for images", Applited Optics, vol. 33, no. 26, (1994).

[14] W. Sweldens, "The lifting scheme: a construction of second generation wavelets", SIAM J. Math. Anal, vol. 29, no. 2, (1998).

[15] X. Wang and G. Yang, "Facial feature extraction based on NSCT and M-PCNN", Computer Engineering, vol. 49, no. 1, (2013).

[16] C. K. Chen and X. M. Gao, "Wavelet Feature-Based Nonlinear Feature Extraction Technique”, Journal of Electronics \& Information Technology, vol. 27, no. 2, (2005).

[17] X. H. Gu, "Visual perception and edge preserving illumination invariant face recognition", Acta Electronica Sinica, vol. 41, no. 8, (2013).

[18] Olivetti \& Oracle Research Laboratory. The Olivetti \& Oracle Research Face Database of Faces[EB/OL].(2010-11-21). http://www.camorl.co.uk/facedatabase.html. 


\section{Authors}

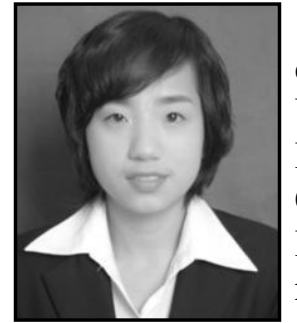

Junhua Liang, she was born in 1985. She received the B.E. degree in Electrical Information Engineering from Qinghai Normal University XiNing China in 2009 and the M.S. degree on Signal and Information Processing from Shaanxi Normal University Xi'An China in 2013. She is currently a teaching assistant in School of Information Science and Engineering at Hebei North University. Her research interests include image processing and pattern recognition.



Zhao Zhisheng, she was born in 1965. She received the M.S. degree on computer application from Hebei University. She is currently professor in School of Information Science and Engineering at Hebei North University. Her research interests include medical data mining, image processing, big data analysis.

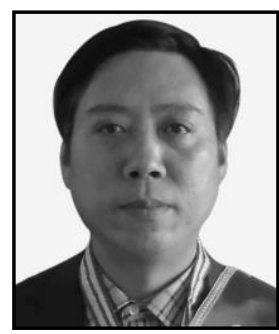

Xiao Zhang, he was born in 1965, and received the M.S. degree on software engineering from East China Normal University in 2012. $\mathrm{He}$ is currently professor and the head of School of Information Science and Engineering at Hebei North University. His research interests include medical information, software engineering, and multimedia development.

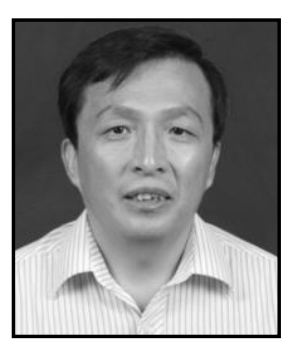

Xuan Wang, he was born in 1966. He received the B.S. and M.S. degrees in Electrical Engineering from Shaanxi Normal University Xi'An, China in 1983 and 1987, and received Ph.D. degree in the key laboratory of the ministry of education for Computer Networks and Information Security. He is currently professor and head of School of Physics and Information Technology at Shaanxi Normal University. His research interests include image processing and pattern recognition.

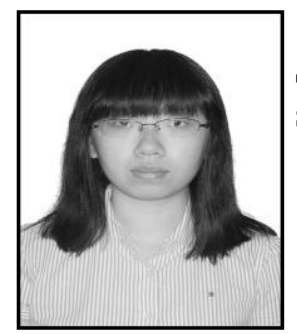

Yang Liu, she is a Lecturer at Hebei North University. Master of Science from University of Missouri.Technical Focus: Computer vision; Machine Learning; Image Processing; Data Mining. 
International Journal of Signal Processing, Image Processing and Pattern Recognition Vol. 9, No. 8 (2016) 